\title{
A construction of hyperbolic Coxeter groups
}

\author{
Damian Osajda*
}

\begin{abstract}
We give a simple construction of Gromov hyperbolic Coxeter groups of arbitrarily large virtual cohomological dimension. Our construction provides new examples of such groups. Using this one can construct e.g. new groups having some interesting asphericity properties.
\end{abstract}

Mathematics Subject Classification (2010). Primary 20F67; Secondary 20F55, 20F65, 51F15, 57M07.

Keywords. Gromov hyperbolic groups, Coxeter groups, systolic groups.

\section{Introduction}

The question of constructing highly dimensional Gromov hyperbolic groups was raised several times in the past. Gromov's loose conjecture [Gro93] (see its precise version on the Bestvina's problem list [Bes]) stated that a construction of such groups always involves nontrivial number theoretic tools (see also the discussion in [JŚ03]). Eventually Januszkiewicz-Świątkowski [JŚ03] provided a geometric construction of Gromov hyperbolic Coxeter groups in every dimension. It should be noticed here that before it was believed; cf. a conjecture of Moussong [Mou88] - that there is a universal bound on the virtual cohomological dimension of any Gromov hyperbolic Coxeter group (this was supported by a result of Vinberg [Vin 85]). Later several similar constructions appeared [Hag03], [JŚ06], [ABJ+09]. All of them are using a fairly advanced machinery of complexes of groups and, moreover, the groups obtained those ways are always systolic in the sense of [JŚ06]. As shown by JanuszkiewiczŚwiątkowski [JŚ07] and the author [Osa07], [Osa08] systolic groups satisfy some very restrictive asphericity properties that make them in a way asymptotically twodimensional. In particular they do not "contain asymptotically" spheres of dimension two and more.

In this paper we give a simple geometric construction of Gromov hyperbolic Coxeter groups of arbitrarily large virtual cohomological dimension. Actually, it is

\footnotetext{
*Partially supported by MNiSW grant N N201 541738 and by the ANR grant "Cannon". This research was supported through the programme "Oberwolfach Leibniz Fellows" by the Mathematisches Forschungsinstitut Oberwolfach in 2010 .
} 
the simplest construction of highly dimensional Gromov hyperbolic groups known to us. Our method is elementary and uses only (in the simplest version) basic facts about right-angled Coxeter groups. It allows us to construct highly dimensional Gromov hyperbolic Coxeter groups that are not systolic - they may contain spheres at infinity. Those are the first examples of this type. On the other hand the general framework presented here allowed us to provide in [OŚ10] new constructions of highly dimensional groups with various asphericity properties. This paper bases on tools and ideas around weakly systolic complexes introduced and developed by the author in [Osa10] (cf. also [CO09]). In particular our construction gives examples of weakly systolic groups of arbitrarily large dimension that are not systolic.

The construction is as follows; $\mathrm{cf}$. the following sections for explanations of all the notions involved. To construct a Gromov hyperbolic right-angled Coxeter group all we need is a finite 5-large (or flag no-square) simplicial complex. We construct such simplicial complexes inductively. Their global cohomological dimension increases each time. The induction step is itself divided into the following three steps.

\section{The basic construction}

Step 1. Let $X$ be a finite 5-large (i.e. flag no-square) simplicial complex with $H^{n}(X ; \mathbb{Q}) \neq 0$. Let $(W, S)$ be the Coxeter system whose nerve is $X$, i.e. $X=$ $L(W, S)$. Then the virtual cohomological dimension of $W$ is vcd $W \geq n+1$. The complex $X$ is the link of every vertex of the Davis complex $\Sigma=\Sigma(W, S)$ of $(W, S)$.

Step 2. Choose a torsion-free subgroup $H$ of sufficiently large finite index in $W$. Then $Y=\Sigma / H$ is a locally 5 -large cubical complex (i.e. with links being flag no-square simplicial complexes) with $H^{n+1}(Y ; \mathbb{Q}) \neq 0$.

Step 3. Let $X^{\prime}=\operatorname{Th}(Y)$ be the simplicial thickening of $Y$ (i.e. the simplicial complex obtained by replacing cubes by simplices spanned on their vertices). Then $X^{\prime}$ is a finite 5-large simplicial complex with $H^{n+1}\left(X^{\prime} ; \mathbb{Q}\right) \neq 0$.

This is the end of the inductive step. The constructed complex $X^{\prime}$ can be now used as the initial complex $X$ again in Step 1. The crucial fact for this construction to work and the main result of the paper is the following.

Main Theorem (cf. Theorem 4.6 in Section 4). Let $X$ be a finite 5-large simplicial complex such that $H^{n}(X ; \mathbb{Q}) \neq 0$. Then there exists a torsion-free finite index subgroup $H$ of $W$ such that the complex $X^{\prime}$ obtained by the basic construction above is a finite 5-large simplicial complex with $H^{n+1}\left(X^{\prime} ; \mathbb{Q}\right) \neq 0$. Thus the right-angled Coxeter group with the nerve $X^{\prime}$ is Gromov hyperbolic of virtual cohomological dimension at least $n+2$. 
Starting with a finite 5-large $n_{0}$-dimensional simplicial complex $X_{0}$, after performing $k$ times Steps 1-3, we get a finite 5-large simplicial complex $X$ with $H^{n_{0}+k}(X ; \mathbb{Q}) \neq 0$. Thus the right-angled Coxeter group with the nerve $X$ is Gromov hyperbolic of virtual cohomological dimension at least $n_{0}+k+1$.

The main idea behind this construction is that one uses the finite quotient $X$ of an " $n$-dimensional" complex (of the Davis complex) as the link of vertices in a new complex (the new Davis complex). Thus the "dimension" of the new complex jumps up by at least one (since the new complex is a union of cones over its links). Then one proceeds inductively.

Organization. Sections 2 and 3 are preliminary sections. In particular, in Section 3 we reprove some technical results from [Osa10] concerning thickenings of cubical complexes. In Section 4 we present in detail the basic construction following the scheme given above. In particular we prove Main Theorem above; cf. Theorem 4.6. Results from Section 5 show that our construction provides examples of Gromov hyperbolic groups of arbitrarily large dimension that are asymptotically different from the groups known before. In particular we show that our groups can "contain asymptotically" spheres of dimension up to 3; cf. Corollary 5.2 and examples afterwards. Finally, in the concluding Section 6 we give some remarks concerning variants of the basic construction.

Acknowledgment. I thank Jan Dymara, Frédéric Haglund, Tadeusz Januszkiewicz and Jacek Świątkowski for helpful conversations.

During writing of this paper I found out that the same idea of the basic construction was discovered independently and at the same time by Frédéric Haglund (unpublished).

\section{Preliminaries}

2.1. Simplicial complexes. Let $X$ be a simplicial complex. The $i$-skeleton of $X$ is denoted by $X^{(i)}$. A subcomplex $Y$ of $X$ is full if every subset $A$ of vertices of $Y$ contained in a simplex of $X$, is contained in a simplex of $Y$. For a finite set $A=\left\{v_{1}, \ldots, v_{k}\right\}$ of vertices of $X$, by $\operatorname{span}(A)$ or by $\left\langle v_{1}, \ldots, v_{k}\right\rangle$ we denote the span of $A$, i.e. the smallest full subcomplex of $X$ containing $A$. A simplicial complex $X$ is flag whenever every finite set of vertices of $X$ joined pairwise by edges in $X$, is contained in a simplex of $X$. The link of a simplex $\sigma$ of $X$ is the simplicial complex $X_{\sigma}=\{\tau \mid \tau \in X, \tau \cap \sigma=\emptyset$ and $\operatorname{span}(\tau \cup \sigma) \in X\}$.

Let $k \geq 4$. The $k$-cycle $\left(v_{0}, \ldots, v_{k-1}, v_{0}\right)$ is the triangulation of a circle consisting of $k$ edges $\left(\left\langle v_{i}, v_{i+1}(\bmod k)\right\rangle\right)$ and $k$ vertices: $v_{0}, \ldots, v_{k-1}$. For $k \geq 4$, a flag simplicial complex $X$ is $k$-large if there are no $j$-cycles being full subcomplexes of $X$, for $j<k$ (4-large means simply flag). In other words it means that for $j<k$ 
each $j$-cycle has a diagonal, i.e. an edge connecting two nonconsecutive vertices. The term flag no square is sometimes used instead of 5-large. A complex is locally $k$-large if all its links are $k$-large. A flag simplicial complex is $k$-systolic, $k \geq 4$, if it is simply connected and locally $k$-large. A group acting geometrically (i.e. properly and cocompactly by automorphisms) on a $k$-systolic complex is called itself $k$-systolic. We use the term systolic as an abbreviation for "6-systolic".

For $i \in \mathbb{N}$, a (combinatorial) ball $B_{i}(v, X)$ of radius $i$ around a vertex $v$ in a simplicial complex $X$, is the full subcomplex spanned by the set of vertices at distance at most $i$ from $v$. Here the distance between two vertices is the minimal number of edges in a 1-skeleton path joining the vertices.

2.2. Cubical complexes. Cubical complexes are cell complexes in which every cell is a cube; see e.g. Appendix A in [Dav08] for a precise definition. It means in particular that two cubes in a cubical complex intersect along a single subcube. The link $Y_{v}$ of a vertex $v$ of a cubical complex $Y$ is the simplicial complex that can be identified with a small sphere around $v$ (simplices of $Y_{v}$ are intersections of the sphere with cubes). A cubical complex is locally $k$-large (resp. locally flag) if links of its vertices are $k$-large (resp. flag). A lemma of Gromov (cf. [Dav08], Appendix I) states that a simply connected locally flag (resp. locally 5-large) cubical complex admits a metric of non-positive (resp. negative) curvature, or in other words a CAT(0) (resp. CAT $(-1))$ metric.

The following result is classical; cf. e.g. [BC08], Section 2.

Helly's Lemma. A finite family of pairwise nontrivially intersecting convex subcomplexes of a $\mathrm{CAT}(0)$ cubical complex has nontrivial intersection.

In particular, any cube and the 1-ball around any vertex (i.e. the union of cubes containing the vertex) are convex.

2.3. Coxeter groups. We use the terminology and notations from the Davis' book [Dav08]. A Coxeter group is given by a presentation $W=\left\langle S \mid(s t)^{m_{s t}} ; s, t \in S\right\rangle$, where $S$ is a finite set, $m_{s t} \in \mathbb{N}^{*} \cup\{\infty\}, m_{s t}=m_{t s}$ and $m_{s t}=1$ iff $t=s$ (here $(s t)^{\infty}$ means no relation). A Coxeter group (or a Coxeter system $(W, S)$ ) is called right-angled if $m_{s t} \in\{1,2, \infty\}$. A special subgroup $W_{T}$ of a Coxeter group $W$ is a subgroup generated by a subset $T \subseteq S$. A subset $T \subseteq S$ is called spherical if $W_{T}$ is finite. In that case $W_{T}$ is called also spherical. By $S$ we denote the poset (w.r.t. inclusions) of spherical subsets of $S$. Its geometric realization is denoted by $K$. The poset of all nonempty spherical subsets is an abstract simplicial complex: the nerve $L=L(W, S)$ of the Coxeter system $(W, S)$. For $T \in S$, by $\sigma_{T}$ we denote the simplex of $L$ spanned by $T\left(\sigma_{T}=\emptyset\right.$ if $\left.T=\emptyset\right)$. The geometric realization of the poset (w.r.t. inclusions) $\bigcup_{T \in \mathcal{S}} W / W_{T}$ is called the Davis complex and is denoted by $\Sigma=\Sigma(W, S)$. In the right-angled case $\Sigma$ possesses a natural structure of a locally 
flag cubical complex. For $s \in S$ we define $K_{s}$ as the union of the simplices in $K$ with minimum vertex $\{s\}$. For $T \subseteq S$ we define $K^{T}=\bigcup_{s \in T} K_{s}$. For $T \in \mathcal{S}$, it can be shown that $L-\sigma_{T}$ deformation retracts onto $K^{S-T}$; cf. [Dav08], Lemma A.5.5.

The lemma of Gromov implies the following.

Theorem 2.1 (Hyperbolic right-angled Coxeter group). A right-angled Coxeter group $(W, S)$ is Gromov hyperbolic iff its nerve $L(W, S)$ is a 5-large (i.e. flag no-square) simplicial complex.

In fact in that case the Davis complex $\Sigma(W, S)$ possesses a natural structure of a locally 5-large (i.e. CAT(-1); cf. Section 2.2) cubical complex.

2.4. Virtual cohomological dimension. Recall (cf. [Dav08], Chapter 8.5) that the cohomological dimension of a group $G$ is defined as

$$
\operatorname{cd} G=\sup \left\{n \mid H^{n}(G ; M) \neq 0 \text { for some } \mathbb{Z} G \text {-module } M\right\} .
$$

If $G$ has nontrivial torsion then $\mathrm{cd} G=\infty$. Thus for virtually torsion-free groups (i.e. groups having a torsion-free subgroup of finite index) the following notion of dimension is more convenient.

The virtual cohomological dimension of a group $G$, denoted vcd $G$, is the cohomological dimension of any torsion-free finite index subgroup of $G$. For a Coxeter system $(W, S)$ as above we have the following (here $\bar{H}^{*}$ denotes the reduced cohomology).

Theorem 2.2 ([Dav08], Corollary 8.5.5).

$$
\begin{aligned}
\operatorname{vcd} W & =\max \left\{n \mid H^{n}\left(K, K^{S-T} ; \mathbb{Z}\right) \neq 0, \text { for some } T \in S\right\} \\
& =\max \left\{n \mid \bar{H}^{n-1}\left(L-\sigma_{T} ; \mathbb{Z}\right) \neq 0, \text { for some } T \in S\right\} .
\end{aligned}
$$

Corollary 2.3. If $\bar{H}^{n-1}(L ; \mathbb{Z}) \neq 0$ then $\operatorname{vcd} W \geq n$.

\section{Thickening of a CAT $(-1)$ cubical complex}

In this section we show how to find, for a given CAT(-1) cubical complex, an associated 5-large (i.e. flag no-square) simplicial complex.

Definition 3.1 (Thickening). Let $Y$ be a cubical complex. The thickening $\operatorname{Th}(Y)$ of $Y$ is a simplicial complex defined in the following way. Vertices of $\operatorname{Th}(Y)$ are vertices of $Y$. Vertices $v_{1}, \ldots, v_{k}$ of $\mathrm{Th}(Y)$ span a simplex iff vertices $v_{1}, \ldots, v_{k} \in Y$ (as vertices of $Y$ ) are contained in a common cube of $Y$. 
The following lemma is proved in [Osa10].

Lemma 3.2 (Locally $k$-large thickening). Let $k \geq 4$ and let $Y$ be a locally $k$-large cubical complex. Then its thickening $\operatorname{Th}(Y)$ is a locally $k$-large simplicial complex.

The proof in the case $k \leq 6$ in the lemma above is much easier than the one in the general case. Moreover, the case $k=5$ is the most interesting for our construction. Thus, for completeness and for the reader's convenience we present it below.

Proof (the case $k=4,5,6$ ). We have to study links of vertices in $\operatorname{Th}(Y)$. Let $v$ be a vertex. Let, for a vertex $w \in \operatorname{Th}(Y)_{v}$, the set $A_{w} \subseteq Y_{v}^{(0)}$ (here we identify the 0 -skeleton of the link of a vertex in a cubical complex with the set of vertices joined with the vertex) be the set of all vertices of $Y_{v}^{(0)} \subseteq Y$ belonging to the minimal cube containing $v$ and $w$.

First we prove that $\operatorname{Th}(Y)_{v}$ is flag. Let $A \subseteq \operatorname{Th}(Y)_{v}^{(0)}$ be a finite set of pairwise connected (by edges in $\operatorname{Th}(Y)_{v}$ ) vertices. Then, by definition of the thickening we have the following. For any two $w, w^{\prime} \in A$, and for every $z \in A_{w}$ and $z^{\prime} \in A_{w^{\prime}}$, vertices $z$ and $z^{\prime}$ are contained in a common cube of $Y$ (the one containing $v, w$, and $\left.w^{\prime}\right)$ and thus $\left\langle z, z^{\prime}\right\rangle \in Y_{v}$. Hence $\bar{A}=\bigcup_{w \in A} A_{w}$ is a set of pairwise connected vertices in $Y_{v}$ and thus, by flagness of $Y_{v}$, it spans a simplex. It follows that $\bar{A} \cup\{v\}$ is contained in a cube of $Y$ so that $A$ is contained in the same cube and thus $A$ spans a simplex in $\operatorname{Th}(Y)_{v}$. It proves that links in $\mathrm{Th}(Y)$ are flag.

Now we prove that $\operatorname{Th}(Y)_{v}$ is $k$-large, for $k=5,6$. We do it by contradiction. Assume there is an $l$-cycle $c=\left(w_{0}, w_{1}, \ldots, w_{l-1}, w_{0}\right)$ in $\operatorname{Th}(Y)_{v}$ without a diagonal, for $l<k$. Then we will show that there exists an $l$-cycle $c^{\prime}=\left(z_{0}, z_{1}, \ldots, z_{l-1}, z_{0}\right)$ in $Y_{v}$ without a diagonal. This would contradict $k$-largeness of $Y_{v}$.

Since there is no diagonal in $c$ we have that for $i \neq j$ and $i \neq j \pm 1(\bmod l)$, there exist vertices $z_{j}^{i} \in A_{w_{i}}$ and $z_{i}^{j} \in A_{w_{j}}$ not contained in a common cube (containing $v$ ) and thus not connected by an edge in $Y_{v}$. Now we treat separately two cases.

(Case $\boldsymbol{l}=4$.) Then the cycle in $Y_{v}$ that leads to a contradiction is

$$
c^{\prime}=\left(z_{2}^{0}, z_{3}^{1}, z_{0}^{2}, z_{1}^{3}, z_{2}^{0}\right) \text {. }
$$

(Case $l=5$.) If $\left\langle z_{2}^{0}, z_{1}^{3}\right\rangle \in Y_{v}$ or $\left\langle z_{3}^{1}, z_{2}^{4}\right\rangle \in Y_{v}$ then, for $c^{\prime}$ we can take, respectively, $\left(z_{2}^{0}, z_{3}^{1}, z_{0}^{2}, z_{1}^{3}, z_{2}^{0}\right)$ or $\left(z_{3}^{1}, z_{4}^{2}, z_{1}^{3}, z_{2}^{4}, z_{3}^{1}\right)$. Assume that it is not true. Then if $\left\langle z_{0}^{2}, z_{2}^{4}\right\rangle \in Y_{v}$, we can set $c^{\prime}=\left(z_{2}^{4}, z_{2}^{0}, z_{3}^{1}, z_{0}^{2}, z_{2}^{4}\right)$, and if not we can take $c^{\prime}=\left(z_{2}^{0}, z_{3}^{1}, z_{0}^{2}, z_{1}^{3}, z_{2}^{4}, z_{2}^{0}\right)$. In every case we get a cycle $c^{\prime}$ without diagonal, of length 4 or 5 .

Now, using the local results above, we prove that in the case of simply connected locally 5-large cubical complexes (i.e. CAT( -1$)$ cubical complexes) their thickenings are 5-large. Results of the following two lemmas were proved in a bigger generality in [Osa10]. 
Lemma 3.3 (Thickening of CAT(0) c.c.). Let $Y$ be a simply connected locally 4-large cubical complex. Then $\operatorname{Th}(Y)$ is a 4-large (i.e. flag) simplicial complex.

Proof. Let $v_{0}, v_{1}, \ldots, v_{k} \in \operatorname{Th}(Y)$ be vertices pairwise joined by edges in $\operatorname{Th}(Y)$. We have to prove that they span a simplex in $\operatorname{Th}(Y)$, that means they are contained (as vertices of $Y$ ) in a common cube.

Let $B_{i}$ be the 1-ball around $v_{i}$ in $Y$, i.e. the union of cubes in $Y$ containing $v_{i}$. Since the balls $B_{i}$ pairwise intersect, by Helly's Lemma there is a vertex $v \in \bigcap_{i} B_{i}$. Since $Y_{v}$ is flag, by Lemma 3.2 we have that $\operatorname{Th}(Y)_{v}$ is flag. Since $v_{0}, v_{1}, \ldots, v_{k} \in$ $B_{1}(v, \operatorname{Th}(Y))$ we get that these vertices span a simplex in $\operatorname{Th}(Y)$.

Proposition 3.4 (Thickening of CAT(-1) c.c.). Let $Y$ be a simply connected locally 5-large cubical complex. Then $\operatorname{Th}(Y)$ is a 5-large simplicial complex.

Proof. By Lemma 3.3 we have that $\operatorname{Th}(Y)$ is flag. Thus it only leaves to show that every 4-cycle in $\operatorname{Th}(Y)$ has a diagonal. Let $\left(v, w, z, w^{\prime}, v\right)$ be a cycle in the 1-skeleton of $\operatorname{Th}(Y)$. Let $c$ and $c^{\prime}$ be cubes containing $z$ and, respectively, $w$ and $w^{\prime}$. Let $B$ be the 1-ball around $v$ in $Y$. By Helly's Lemma there exists a vertex $u \in c \cap c^{\prime} \cap B$. Hence we have that $v, w, w^{\prime}, z \in \operatorname{Th}(Y)_{u}$ and, by Lemma 3.2, we have that the cycle $\left(v, w, z, w^{\prime}, v\right)$ has a diagonal since $\operatorname{Th}(Y)$ is locally 5-large.

Lemma 3.5. Let $Y$ be a finite dimensional cubical complex. Then $\operatorname{Th}(Y)$ is homotopically equivalent to $Y$.

Proof. Let $\mathcal{N}$ be the nerve of the cover of $Y$ by (closed) cubes. Then, by the Nerve Theorem of Borsuk [Bor48], $\mathcal{N}$ is homotopically equivalent to $Y$. Since $\mathcal{N}$ is at the same time the nerve of the cover of $\operatorname{Th}(Y)$ by (closed) simplices, again by the Nerve Theorem we get the assertion of the lemma.

Lemma 3.6. Let $Y$ be a locally $k$-large, simply connected cubical complex, for some $k \geq 4$. Then $\operatorname{Th}(Y)$ is a $k$-systolic, contractible simplicial complex.

Proof. By Lemma 3.2, Th( $Y)$ is locally $k$-large, and by Lemma 3.5, it is contractible (since CAT $(0)$ cubical complexes are contractible).

For a cubical complex $Y$ we can identify links of vertices in $Y$ with subcomplexes in links of vertices of $\operatorname{Th}(Y)$. The following lemma is obvious. It will be used in Section 5 .

Lemma 3.7. Let $v$ be a vertex of a locally flag cubical complex $Y$. Then $Y_{v}$ is a full subcomplex of $\operatorname{Th}(Y)_{v}$, and hence every full subcomplex of $Y_{v}$ is a full subcomplex of $\operatorname{Th}(Y)_{v}$. 
Remark. In the more general setting of cell complexes, the thickening was invented by T. Januszkiewicz and, independently, by the author; cf. [Osa10]. In the case of cubical complexes, a construction similar to the thickening has been used in graph theory. For a graph $G$ being the 1-skeleton of a CAT(0) cubical complex $Y$ (such graphs are called median graphs), a graph $G^{\Delta}$ is defined as the 1-skeleton of $\operatorname{Th}(Y)$; cf. [BC08], Section 3.1 (we use their notation here).

\section{The basic construction}

In this section we present in detail the construction of high dimensional Gromov hyperbolic right-angled Coxeter groups as described roughly in the introduction. We will keep here the notations introduced there.

By Theorem 2.1, a Gromov hyperbolic right-angled Coxeter group $W$ is determined by its nerve $L=L(W, S)$ (where $S$ is a given set of generators of $W$ ) that is itself a finite 5-large simplicial complex. By Corollary 2.3, the virtual cohomological dimension of $W$ is estimated by the global cohomological dimension of $L$. Thus the question reduces to a construction of finite 5-large simplicial complexes $X$ of arbitrarily large global cohomological dimension, i.e. with $H^{n}(X ; \mathbb{Z}) \neq 0$ for large $n$.

We construct such complexes by induction. Their "dimension" will increase at each step.

The base of the induction is a finite 5-large simplicial complex $X_{0}$ satisfying $H^{n_{0}}\left(X_{0}, \mathbb{Q}\right) \neq 0$, for some $n_{0} \geq 1$. As an example one can choose $X_{0}$ to be a finite graph of girth at least 5 (i.e. not containing cycles of length less than 5 ) containing a $k$-cycle for some $k \geq 5$.

The induction step is as follows. The input data is a finite 5-large simplicial complex $X$ with $H^{n}(X ; \mathbb{Q}) \neq 0$, for $n \geq n_{0}$. As an output we obtain a finite 5-large simplicial complex $X^{\prime}$ with $H^{n+1}\left(X^{\prime} ; \mathbb{Q}\right) \neq 0$.

Now we present the induction step in detail following the overview given in Introduction. It consists itself of the three following steps.

Step 1. Let $(W, S)$ be the right-angled Coxeter system whose nerve is $X$, i.e. $X=$ $L(W, S)$. The Davis complex $\Sigma=\Sigma(W, S)$ is a contractible cubical complex in which links of all vertices are isomorphic to $X$. Thus it is a contractible locally 5-large cubical complex.

Step 2. In this step we find a torsion-free subgroup $H<W$ of large finite index such that $Y=H \backslash \Sigma$ is a locally 5-large cubical complex of rational global cohomological dimension at least $n+1$, i.e. with $H^{n+1}(Y ; \mathbb{Q}) \neq 0$. The only difficulty here is to prove the last inequality. We do it, using standard tools (cf. [Dav08]), by constructing a nontrivial cohomology class in $H^{n+1}(Y ; \mathbb{Q})$; see Lemma 4.5 below. 
We use simplicial cohomology w.r.t. the standard triangulation of $\Sigma$, in which the Davis chamber $K$ is the cone over the barycentric subdivision of the nerve $L$.

For $H<W$, let $Y=H \backslash \Sigma$ and let $p: \Sigma \rightarrow Y$ be the quotient map. Let $K$ denote the chamber corresponding to the coset of identity in $\Sigma$ and let $\mathcal{K}$ be the set of copies of $K$ in $Y$, i.e. $\mathcal{K}=\{p(w K) \mid w \in W\}$.

Definition 4.1 (Orientation). An orientation of $Y=H \backslash \Sigma$ is a map $\epsilon: \mathcal{K} \rightarrow\{-1,1\}$ such that $\epsilon(p(w s K))=-\epsilon(p(w K))$, for every $w \in W$ and $s \in S$.

Observe that $\epsilon(w K)=(-1)^{l_{S}(w)}$ (here $l_{S}(w)$ denotes the length of $w$ in the word metric on $W$ w.r.t. $S$ ) defines an orientation on $Y=\Sigma$, i.e. in the case $H=\{1\}$.

By Corollary D.1.4 of [Dav08], Coxeter groups are virtually torsion-free and thus there exists a finite index torsion-free subgroup $H$ of $W$. Coxeter groups are residually finite (see e.g. [Dav08], Section 14.1) so that we can choose $H$ whose minimal displacement is "big". For our purposes we make the following choice. We take a torsion-free subgroup $H<W$ such that for the action (induced by the $W$-action on $\Sigma$ ) of $H$ on $\operatorname{Th}(\Sigma)$ (on the thickening of $\Sigma$; cf. Section 3) we have $\inf \left\{d(v, h v) \mid v \in \operatorname{Th}(\Sigma)^{(0)}, h \in H\right\} \geq 5$, where $d(\cdot, \cdot)$ is the usual metric on $\operatorname{Th}(\Sigma)^{(1)}$.

As in the case of manifolds which have two-sheeted orientable covering, the same is true here, with an analogous proof.

Lemma 4.2. There exists a covering of $Y$ of degree at most two, which admits an orientation.

Thus wlog we can assume that there is an orientation $\epsilon: \mathcal{K} \rightarrow\{-1,1\}$ of $Y$. From now on we fix an orientation $\epsilon$ for the rest of this section. We get an orientation $\epsilon^{\prime}$ of $\Sigma$ by setting $\epsilon^{\prime}(w(K))=\epsilon(p(w K))$. We will write $\epsilon$ instead of $\epsilon^{\prime}-$ it should not lead to misunderstanding.

Below we introduce an operation of antisymmetrization of cochains on $Y$. It produces a cochain whose value on a simplex $\sigma$ inside $K$ is a signed (w.r.t. the orientation $\epsilon$ ) average over all simplices "congruent" to $\sigma$ - one for each copy of $K$ in $Y$.

Definition 4.3 (Antisymmetrization of cochains). For $h \in C^{*}(Y ; \mathbb{Q})$, the antisymmetrization of a cochain $h$ is a cochain $a(h)$ defined as follows:

$$
a(h)(p(w \sigma))=\frac{\epsilon(w K)}{|\mathcal{K}|} \sum_{K^{\prime} \in \mathcal{K}} \epsilon\left(K^{\prime}\right) h\left(p\left(w_{K^{\prime}} \sigma\right)\right),
$$

where $w \in W, \sigma$ is a simplex in $K, w_{K^{\prime}} \in W$ and $p\left(w_{K^{\prime}} \sigma\right) \in K^{\prime}$. 
It is clear that the cochain obtained by antisymmetrization attains the same absolute value on "congruent" simplices and its sign changes on "s-adjacent" $(s \in S)$ simplices. In the following lemma we show precisely the properties of antisymmetrization that we need later.

Lemma 4.4 (Properties of $a(h)$ ). Let $h \in C^{*}(Y ; \mathbb{Q})$. The antisymmetrization has the following properties:

(1) $\delta a(h)=a(\delta h)$,

(2) $a(a(h))=a(h)$.

Proof. Let $w \in W$ and let $\sigma$ be a simplex in $K$. Then we have the following.

(1)

$$
\begin{aligned}
\delta a(h)(p(w \sigma)) & =a(h)(\partial p(w \sigma))=a(h)(p(w \partial \sigma)) \\
& =\frac{\epsilon(w K)}{|\mathcal{K}|} \sum_{K^{\prime} \in \mathcal{K}} \epsilon\left(K^{\prime}\right) h\left(\partial\left(p\left(w_{K^{\prime}} \sigma\right)\right)\right) \\
& =\frac{\epsilon(w K)}{|\mathcal{K}|} \sum_{K^{\prime} \in \mathcal{K}} \epsilon\left(K^{\prime}\right) \delta h\left(p\left(w_{K^{\prime}} \sigma\right)\right) \\
& =a(\delta h)(p(w \sigma)) .
\end{aligned}
$$

(2)

$$
\begin{aligned}
a(a(h))(p(w \sigma)) & =\frac{\epsilon(w K)}{|\mathcal{K}|} \sum_{K^{\prime} \in \mathcal{K}} \epsilon\left(K^{\prime}\right) a(h)\left(p\left(w_{K^{\prime}} \sigma\right)\right) \\
& =\frac{\epsilon(w K)}{|\mathcal{K}|} \sum_{K^{\prime \prime} \in \mathcal{K}} \epsilon\left(K^{\prime \prime}\right) h\left(p\left(w_{K^{\prime \prime}} \sigma\right)\right) \sum_{K^{\prime} \in \mathcal{K}} \epsilon\left(K^{\prime}\right) \frac{\epsilon\left(K^{\prime}\right)}{|\mathcal{K}|} \\
& =a(h)(p(w \sigma)) .
\end{aligned}
$$

By our inductive assumptions, there exists a cocycle $f \in Z^{n+1}\left(K, K^{S} ; \mathbb{Q}\right)$ representing a nontrivial class $[f] \in H^{n+1}\left(K, K^{S} ; \mathbb{Q}\right)=H^{n}(X ; \mathbb{Q})$. We can "extend" $f$ to the cochain on $Y$ by setting: $f(p(\sigma))=f(\sigma)$ for $\sigma$ in $K$, and $f(p(\sigma))=0$ if $p(\sigma)$ is not in $p(K)$. Define a cochain $f^{\prime}=|\mathcal{K}| a(f)$ on $Y$.

The $(n+1)$-cochain $f^{\prime}$ is the antisymmetrization of the "radial" extension of the nontrivial $n$-cochain $f \in Z^{n}(X ; \mathbb{Q})$. The next crucial lemma shows that $f^{\prime}$ represents a nontrivial cohomology class $\left[f^{\prime}\right]$. The geometric picture behind the proof is as follows. If $\left[f^{\prime}\right]$ is trivial then $f^{\prime}$ is a coboundary of an "antisymmetric" $n$ cochain $a\left(g^{\prime}\right)$, i.e. $a\left(g^{\prime}\right)$ vanishes on simplices in the "boundary" $K^{S}$ of the chamber $K$. Thus the restriction $g$ of $a\left(g^{\prime}\right)$ to $K$ is the cochain whose coboundary is $f$, contradicting nontriviality of $[f]$. 
Lemma 4.5 (Nontriviality of $\left[f^{\prime}\right]$ ). The cochain $f^{\prime}$ is a cocycle representing a nontrivial class $\left[f^{\prime}\right] \in H^{n+1}(Y ; \mathbb{Q})$.

Proof. First we prove that $\delta f^{\prime}=0$. This follows from Lemma 4.4, since $\delta f^{\prime}=$ $\delta|\mathcal{K}| a(f)=|\mathcal{K}| a(\delta f)=0$ (the last equality follows from the fact that $f$ vanishes on $K^{S}$ ).

Now we prove that $\left[f^{\prime}\right] \neq 0$ in $H^{n+1}(Y ; \mathbb{Q})$. Suppose this is not so. Let $f^{\prime}=\delta g^{\prime}$ for some cochain $g^{\prime} \in C^{n}(Y ; \mathbb{Q})$. Then, by Lemma 4.4, $\delta a\left(g^{\prime}\right)=a\left(\delta g^{\prime}\right)=a\left(f^{\prime}\right)=$ $a(|\mathcal{K}| a(f))=f^{\prime}$. Observe that $a\left(g^{\prime}\right)$ vanishes on $p\left(w K^{S}\right)$, for every $w \in W$. Let $g \in C^{n}\left(K, K^{S} ; \mathbb{Q}\right)$ be defined as $g(\sigma)=a\left(g^{\prime}\right)(p(\sigma))$, for $\sigma$ in $K$. Then, for any $(n+1)$-simplex $\sigma$ in $K$, we have

$$
\begin{aligned}
\delta g(\sigma) & =a\left(g^{\prime}\right)(p(\partial \sigma))=\frac{\epsilon(K)}{|\mathcal{K}|} \sum_{K^{\prime} \in \mathcal{K}} \epsilon\left(K^{\prime}\right) g^{\prime}\left(\partial\left(p\left(w_{K^{\prime}} \sigma\right)\right)\right) \\
& =\frac{\epsilon(K)}{|\mathcal{K}|} \sum_{K^{\prime} \in \mathcal{K}} \epsilon\left(K^{\prime}\right) f^{\prime}\left(p\left(w_{K^{\prime}} \sigma\right)\right) \\
& =\frac{\epsilon(K)}{|\mathcal{K}|} \sum_{K^{\prime} \in \mathcal{K}} \epsilon\left(K^{\prime}\right)|\mathcal{K}| \frac{\epsilon\left(K^{\prime}\right)}{|\mathcal{K}|} \sum_{K^{\prime \prime} \in \mathcal{K}} \epsilon\left(K^{\prime \prime}\right) f\left(p\left(w_{K^{\prime \prime}} \sigma\right)\right) \\
& =\epsilon(K) \sum_{K^{\prime \prime} \in \mathcal{K}} \epsilon\left(K^{\prime \prime}\right) f\left(p\left(w_{K^{\prime \prime}} \sigma\right)\right)=f(\sigma) .
\end{aligned}
$$

This contradicts the fact that $[f] \neq 0$ in $H^{n+1}\left(K, K^{S} ; \mathbb{Q}\right)$ and finishes the proof of the lemma.

Step 3. Let $X^{\prime}=\operatorname{Th}(Y)$. Then, by Lemma 3.5 and Lemma $4.5, H^{n+1}\left(X^{\prime} ; \mathbb{Q}\right)=$ $H^{n+1}(Y ; \mathbb{Q}) \neq 0$. Observe that $X^{\prime}=H \backslash \mathrm{Th}(\Sigma)$. Since, by Lemma 3.4, the complex $\operatorname{Th}(\Sigma)$ is 5-large, our choice of $H$ (with large minimal displacement) guarantees that $X^{\prime}$ is 5-large.

Let us conclude what we get in Steps 1-3, in the following theorem; cf. Main Theorem from Introduction.

Theorem 4.6. Let $X$ be a finite 5-large simplicial complex such that $H^{n}(X ; \mathbb{Q}) \neq 0$. Then the complex $X^{\prime}$ (as obtained in Steps 1-3) is a finite 5-large simplicial complex and $H^{n+1}\left(X^{\prime} ; \mathbb{Q}\right) \neq 0$. Thus $H^{n+1}\left(X^{\prime} ; \mathbb{Z}\right) \neq 0$ and the right-angled Coxeter group with nerve $X^{\prime}$ is Gromov hyperbolic of virtual cohomological dimension at least $n+2$.

\section{New examples of hyperbolic Coxeter groups}

In this section we show that our construction provides also examples of highly dimensional Gromov hyperbolic Coxeter groups that are very (i.e. asymptotically) different 
from the ones constructed before. In fact, all such Coxeter groups obtained by using constructions from [JŚ03], [JŚ06], [Hag03], [ABJ+09] are systolic. Systolic groups are in a way two-dimensional - they "do not contain asymptotically" spheres above dimension one; cf. [JŚ07], [Osa07], [Osa08], [OŚ10]. We show how to get non-systolic examples (cf. Corollary 5.2 and examples afterwards) - they "contain" spheres up to dimension 3 .

Proposition 5.1 (Subgroups). Let $Z$ be a full subcomplex of a finite 5-large simplicial complex $X_{0}$. Let $X$ be a finite 5-large simplicial complex obtained by the basic construction starting with $X_{0}$ (i.e. by performing several times Steps 1-3 from Section 4$)$ and let $(W, S)$ be the resulting Coxeter system (i.e. $X=L(W, S)$ ). Then the right-angled Coxeter group $W^{\prime}$ with the nerve $Z$ (i.e. $Z=L\left(W^{\prime}, S^{\prime}\right)$ ) is a subgroup of $W$.

Proof. By repeated use of Lemma 3.7 and the fact that a vertex link of a flag simplicial complex is a full subcomplex, we get that $Z$ is a full subcomplex of $X$. Thus it corresponds to the subgroup $W^{\prime}$ of $W$; cf. Section 8.8 in [Dav08].

For distinguishing some of "our" groups from systolic groups we use the notion of asymptotic hereditary asphericity (shortly AHA), which we do not define here see [JŚ07], [OŚ10] for details. AHA is a rigorous way of saying that a group "does not contain asymptotically" spheres.

Corollary 5.2 (Non-systolic). If $X_{0}$ is the nerve of a group $W^{\prime}$ which is not asymptotically hereditarily aspherical, then a group $W$ obtained by the basic construction is not systolic.

Proof. It follows directly from Proposition 5.1 above and from the fact that subgroups of systolic groups are AHA; cf. [JŚ07], [OŚ10].

Examples. Here we show how our construction can lead to non-systolic examples. Let $X_{0}$ be the nerve of a right-angled Coxeter group $W^{\prime}$ acting geometrically on the $k$-dimensional real hyperbolic space $\mathbb{H}^{k}$ (such groups exist only for $k=1,2,3,4$; cf. [Vin 85]). If $k \geq 3$, then $W^{\prime}$ is not AHA (cf. [JŚ07], [Osa07], [OŚ10]) and thus $W$ (a group obtained by our construction) is not AHA, in particular not systolic; cf. [JŚ07], [OŚ10].

Remark. We do not know whether Gromov hyperbolic right-angled Coxeter group can "contain asymptotically" spheres above dimension 3 . A (form of a) conjecture by Januszkiewicz-Świątkowski says that the Gromov boundary of a simply connected locally 5-large cubical complex (i.e. CAT( -1$)$ cubical complex) cannot contain spheres of dimension above 3. Since hyperbolic right-angled Coxeter groups act geometrically on such cubical complexes (their Davis complexes), this conjecture implies that their boundaries cannot contain highly dimensional spheres. 


\section{Final remarks}

6.1. Systolic groups and groups with various asphericity properties. In contrast to the results in Section 5 here we present the following proposition, whose immediate proof using Lemma 3.6 is left to the reader.

Proposition 6.1 (Systolic groups). Let $k \geq 6$ and let $X$ be a finite 5-large simplicial complex obtained by the basic construction (as in Section 4). Assume that the two following conditions are satisfied.

1) The initial complex $X_{0}$ is $k$-large (cf. Section 4).

2) At every step of the basic construction the group $H$ (cf. Step 2 in Section 4) is chosen so that the minimal displacement of the H-action on $\operatorname{Th}(\Sigma)^{(1)}$ is at least $k$.

Then the resulting complex $X$ is $k$-large and, consequently, the Coxeter group $W$ with the nerve $X$ is $k$-systolic.

Moreover, other variants of the basic construction are used in [OŚ10] to obtain new constructions of groups with various asphericity properties satisfied by systolic groups; cf. [JŚ07], [Osa07], [Osa08]. This relies on the notion of weakly systolic groups introduced recently by the author; cf. [Osa10], [CO09].

6.2. Variants of the basic construction. There are many variants of the basic construction corresponding to the choice of the complex $Z$ playing the role of $\Sigma$ in Step 1 (cf. Section 4), for a fixed $X$. Let us describe here one of them.

For a given $X, W$ and $\Sigma$ as in Step 1 of the basic construction, let $Z$ be a regular right-angled building associated with $W$. A simple construction of such buildings is described in [DO07]. It is also showed there (reproving known facts in a new way) that groups of isometries of such buildings admit residually finite uniform lattices. In [Osa10] it is shown that for any such building there exists a locally 5-large simplicial complex $\operatorname{Th}(Z)$, being an analogue of the thickening of a cubical complex described in Section 3: simplices of $\operatorname{Th}(Z)$ correspond to spherical residues in $Z$. Thus, for an appropriate $H$ being a lattice in $\operatorname{Isom}(Z)$, the quotient $H \backslash \operatorname{Th}(Z)$ is a finite 5-large simplicial complex with $H^{n+1}(H \backslash \operatorname{Th}(Z)) \neq 0$ (since $Z$ contains copies of $\Sigma$ ). And thus we can continue the induction.

6.3. Non right-angled case. Given a Gromov hyperbolic right-angled Coxeter group $W$ with a presentation $W=\left\langle S \mid(s t)^{m_{s t}}\right\rangle$, one can construct a non right-angled Coxeter group $W^{f}=\left\langle S \mid(s t)^{m_{s t}^{f}}\right\rangle$ as follows. If $m_{s t} \neq \infty$, then $m_{s t}^{f}=m_{s t}$, otherwise $m_{s t}^{f}$ is an arbitrary number larger than 4 , or $\infty$. It is proved in [JŚ03] that $W^{f}$ is then Gromov hyperbolic and that $\operatorname{vcd}\left(W^{f}\right)=\operatorname{vcd}(W)$, if $\operatorname{vcd}(W) \geq 2$. 


\section{References}

[ABJ+09] G. Arzhantseva, M. R. Bridson, T. Januszkiewicz, I. J. Leary, A. Minasyan and J. Świątkowski, Infinite groups with fixed point properties. Geom. Topol. 13 (2009), no. 3, 1229-1263. Zbl 1197.20034 MR 2496045

[BC08] H.-J. Bandelt and V. Chepoi, Metric graph theory and geometry: a survey. In Surveys on discrete and computational geometry, Contemp. Math. 453, Amer. Math. Soc., Providence, RI, 2008, 49-86. Zbl 1169.05015 MR 2405677

[Bes] M. Bestvina, Questions in geometric group theory. http://www.math.utah.edu/ bestvina/eprints/questions-updated.pdf

[Bor48] K. Borsuk, On the imbedding of systems of compacta in simplicial complexes. Fund. Math. 35 (1948), 217-234. Zbl 0032.12303 MR 0028019

[CO09] V. Chepoi and D. Osajda, Dismantlability of weakly systolic complexes and applications. Preprint, arXiv:0910.5444v1.

[Dav08] M. W. Davis, The geometry and topology of Coxeter groups. London Math. Soc. Monogr. Ser. 32, Princeton University Press, Princeton, NJ, 2008. Zbl 1142.20020 MR 2360474

[DO07] J. Dymara and D. Osajda, Boundaries of right-angled hyperbolic buildings. Fund. Math. 197 (2007), 123-165. Zbl 1177.20042 MR 2365885

[Gro93] M. Gromov, Asymptotic invariants of infinite groups. Geometric group theory, Vol. 2, London Math. Soc. Lecture Note Ser. 182, Cambridge University Press, Cambridge 1993, 1-295. Zbl 0841.20039 MR 1253544

[Hag03] F. Haglund, Complexes simpliciaux hyperboliques de grande dimension. Prepublication Orsay 71, 2003.

http://www.math.u-psud.fr/ biblio/ppo/2003/fic/ppo_2003_71.pdf

[JŚ03] T. Januszkiewicz and J. Świątkowski, Hyperbolic Coxeter groups of large dimension. Comment. Math. Helv. 78 (2003), no. 3, 555-583. Zbl 1068.20043 MR 1998394

[JŚ06] T. Januszkiewicz and J. Świątkowski, Simplicial nonpositive curvature. Publ. Math. Inst. Hautes Études Sci. No. 104 (2006), 1-85. Zbl 1143.53039 MR 2264834

[JŚ07] T. Januszkiewicz and J. Świątkowski, Filling invariants of systolic complexes and groups. Geom. Topol. 11 (2007), 727-758. Zbl 1188.20043 MR 2302501

[Mou88] G. Moussong, Hyperbolic Coxeter groups. Ph.D. thesis, The Ohio State University, 1988.

[Osa07] D. Osajda, Connectedness at infinity of systolic complexes and groups. Groups Geom. Dyn. 1 (2007), no. 2, 183-203. Zbl 1128.20029 MR 2319456

[Osa08] D. Osajda, Ideal boundary of 7-systolic complexes and groups. Algebr. Geom. Topol. 8 (2008), no. 1, 81-99. Zbl 1147.20037 MR 2377278

[Osa10] D. Osajda, A combinatorial non-positive curvature I: weak systolicity. In preparation.

[OŚ10] D. Osajda and J. Świątkowski, On asymptotically hereditarily aspherical groups. Preprint, available at http://www.math.uni.wroc.pl/ dosaj/

[Vin 85] È. B. Vinberg, Hyperbolic groups of reflections. Uspekhi Mat. Nauk 40 (1985), no. 1 (241), 29-66, 255; English transl. Russ. Math. Surv. 40 (1985), no. 1, 31-75. Zbl 0579.51015 MR 0783604 
Received March 3, 2010

Damian Osajda, Universität Wien, Fakultät für Mathematik, Nordbergstraße 15, 1090 Wien, Austria; and (on leave) Instytut Matematyczny, Uniwersytet Wrocławski, pl. Grunwaldzki 2/4, 50-384 Wrocław, Poland

E-mail: dosaj@math.uni.wroc.pl 